\title{
QoS Oriented and Energy Efficient Routing Protocol for Cooperative MIMO Based Mobile WSN: Q-E2RPC
}

\author{
Kummathi Chenna Reddy ${ }^{1, *}$, Geetha D Devanagavi ${ }^{1}$ and Thippeswamy $\mathrm{MN}^{2}$ \\ ${ }^{I}$ School of Electronics and Communication, REVA University, Bangalore, India \\ ${ }^{2}$ Department of Computer Science, Nitte Meenakshi Institute of Technology, Bangalore, India
}

Received 3 September 2018; Accepted 2 January 2019

\begin{abstract}
In this paper, a robust mobility assisted WSN routing protocol named QoS oriented and Energy Efficient Routing Protocol for Cooperative Multiple Input Multiple Output (MIMO) based mobile WSNs (Q-E2RPC) has been developed that exploits the efficiency of network partitioning, Fuzzy Clustering Mean (FCM) and Expectation Maximization (EM) based clustering, Fuzzy Logic Controller (FLC) based Cluster Head (CH) selection and mobile sink based data gathering to meet QoS demands and energy efficiency. Unlike classical clustering methods FCM and EM as a cumulative solution enables optimal clustering, which is followed by the multiple network parameters based $\mathrm{CH}$ selection. The use of single mobile sink avoided multi-hop transmission and signaling overheads that eventually reduced energy consumption. QE2RPC protocol exhibited timely data delivery, low energy consumption, and reduced signaling overheads. The results exhibited that Q-E2RPC outperforms other state-of-art techniques in terms of higher throughput, low delay and energy consumption, and higher network efficacy.
\end{abstract}

Keywords: Wireless Sensor Networks; Cooperative MIMO; Fuzzy Logic Controller; Expectation Maximization.

\section{Introduction}

Wireless sensor network (WSN) has emerged as one of the most efficient network solutions. The robustness of WSN makes it suitable for being used in major applications, including healthcare, civil surveillance, defense systems, industrial monitoring and control, business communication, traffic surveillance and control, and critical data sensing and control [1]. WSN has gained significant attention to enable low cost communication solution for Internet of Things (IoT) ecosystem, primarily in Low Power Lossy Network (LLNs) [2]. To fulfill quality of service (QoS) demands and energy efficient communication, numerous efforts have been made [3-6], where emphasis is made on achieving higher bandwidth utilization, minimal end-to-end delay, minimum data loss and energy consumption, reliable communication etc. However, limited energy and continuous sensory communication forces WSN to undergo exhaustion and network or node-dead condition. Among major solutions cooperative communication has been found potential to support efficient routing. Sharing of real time network statistics among the connected nodes (CNs) helps in better routing decision. To assist energy and resource efficient communication, Multiple-Input Multiple-Output (MIMO) technique is found potential [7]. It plays vital role in alleviating the key issues of low transmission rates and low reliability. Since, the lifetime of each sensor node primarily relies on its battery capacity; WSNs require dealing with energy exhaustion issues. Constructing WSN nodes having

*E-mail address: chenna.cr@gmail.com

ISSN: 1791-2377 @ 2019 Eastern Macedonia and Thrace Institute of Technology. All rights reserved. doi:10.25103/jestr.121.25 multiple antennas could be difficult because of the size and complexity related constraints.

To deal with this situation, WSNs can apply MIMO in cooperative way that can assure reliable communication but also energy efficient transmission over defined network region. In CMIMO multiple nodes construct transmission and receiving clusters by performing synchronization and synchronize data exchange to ensure that the clusters could apply standard MIMO for communication. CMIMO exploits the cooperative nature of the densely deployed nodes to ensure reliable communication. It intends to reduce energy consumption and delay while enabling higher throughput at the receiver to meet QoS provision. CMIMO using multiple sensor nodes functional in cooperative manner makes communication more efficient. In this technique, multiple sensor nodes are physically connected or clustered to perform communication, where within a cluster, sensor nodes communicate with relatively low power than to intergroup communication. MIMO system also requires performing early (i.e., delay sensitive) data gathering to alleviate the issue of redundancy and energy exhaustion. Typically, data redundancy is caused because of relaying the signal to nearby users working as the intermediate nodes. CMIMO for WSN discriminates itself in terms of data redundancy from user CMIMO systems [8-10]. Clustering based WSN routing protocol encompasses node grouping and $\mathrm{CH}$ selection, which is performed in such a manner that the non- $\mathrm{CH}$ nodes could communicate with connected $\mathrm{CH}$ directly. In CMIMO based WSN, $\mathrm{CH}$ forwards gathered data to the sink directly or in multi-hop paradigm. Thus, the groups of $\mathrm{CHs}$ constitute a connected dominating set. However, factors like the optimal number of clusters, optimal $\mathrm{CH}$ selection, and transmission play decisive role in WSN performance. Unlike generic multi-hop transmission 
Kummathi Chenna Reddy, Geetha D Devanagavi and Thippeswamy MN/

Journal of Engineering Science and Technology Review 12 (1) (2019) 213 - 224

based WSNs, the use of a single mobile sink can avoid unwanted retransmission and hence can assure higher throughput, low energy consumption and delay. With this motivation, in this paper the emphasis is made on applying an enhanced grid partitioning, hybrid (or dual phase) clustering, multi-parameters (distance, RSSI, responsiveness) based $\mathrm{CH}$ selection, cooperative MIMO based transmission, and mobile sink based data collection to meet QoS demands and energy efficient communication over WSNs. The results obtained have exhibited that the proposed QoS Oriented Energy Efficient Routing Protocol for CMIMO based Mobile-WSN (Q-E2RPC) outperforms existing state of art techniques.

Considering the significance of QoS oriented and energy efficient WSN routing protocol, this study intends to incorporate a multi-objective optimization measure to improve node clustering, $\mathrm{CH}$ selection, and data transmission. Q-E2RPC model exploits both FCM and EM based two-phased clustering, FLC based $\mathrm{CH}$ selection, CMIMO based transmission and single mobile sink based data collection. Our overall research effort intends to retrieve answers for the following questions Guidelines:

1. Can multiple network parameters (i.e., residual energy, link quality or SNR, distance, node responsiveness etc) as cumulative network statistics be significant to enable efficient $\mathrm{CH}$ selection?

2. Can the use of soft computing algorithm such as Fuzzy Logic Controller be efficient to perform clustering and $\mathrm{CH}$ selection?

3. Can fuzzy logic be significant to perform multiple network parameter based CH selection to assist CMIMO based transmission over WSNs?

4. Can Expectation Maximization approach be efficient to strengthen FCM based clustering to achieve reliable and efficient communication over large scale WSNs?

5. Can the use of Mobile Sink be a plus to assist timely and energy efficient communication over CMIMO based WSNs?

6. Can the above mentioned contributions (1-5) be effective to enable a QoS oriented and energy efficient routing protocol for WSNs?

The list of abbreviations and respective definition is given in Table 1.

Table 1. List of Abbreviation

\begin{tabular}{l|l}
\hline Abbreviation & \multicolumn{1}{c}{ Definition } \\
\hline QoS & Quality of Service \\
MIMO & Multiple Input Multiple Output \\
Q-E2RPC & QoS oriented and Energy Efficient \\
& Routing Protocol for Cooperative Multiple \\
& $\begin{array}{l}\text { Input Multiple Output (MIMO) based } \\
\text { mobile WSNs }\end{array}$ \\
WSNs & Wireless Sensor Networks \\
FCM & Fuzzy Clustering Mean \\
FLC & Fuzzy logic controller \\
EM & Expectation Maximization \\
CH & Cluster Head \\
IoT & Internet of Things \\
LLNs & Low Power Lossy Network \\
CNs & connected nodes \\
CMIMO & Cooperative Multiple Input Multiple \\
RSSI & Output \\
LEACH & Received Signal Strength Indicator \\
& Low-Energy Adaptive Clustering
\end{tabular}

\begin{tabular}{l|l} 
& Hierarchy \\
MAC & medium access control \\
EE-LEACH & energy-efficient LEACH \\
MCMIMO & Multi-Channel CMIMO \\
VMIMO & Virtual MIMO \\
CCP & Cooperative Clustering Protocol \\
2D & Two dimensional \\
SIMO & Single Input Multiple Output \\
MISO & Multiple Input Single Output \\
STBC & Space-Time Block Coding \\
CH-C-TEEM & CH Cooperative Trustworthy Energy \\
& Efficient MIMO \\
EBCS & Energy Based Clustering Self organizing \\
& map \\
EMHR-FL & Multi-hop Hierarchical Routing Protocol \\
& using Fuzzy Logic \\
FIS & Fuzzy Logic Inference System \\
SNR & Signal to Noise Ratio \\
CSI & Channel State Information \\
CM & Covariance Matrix \\
DoD & Degree of Dependence \\
CoG & Center of Gravity \\
TSP & Traveling Salesman Problem \\
DTR & Data Transmission Request \\
\hline
\end{tabular}

\section{Related Work}

This section primarily discusses some of the key literatures presenting WSN routing protocol, particularly using CMIMO technique. He et al. [11] developed CMIMO model by applying clusters of different sizes. Authors found that with clusters of the same size, $\mathrm{CH}$ with relatively lower residual energy might undergo fast energy exhaustion than the one with more residual energy. This as a result may provoke reclustering iteratively for $\mathrm{CH}$ selection and therefore may consume more energy. To deal with it, authors applied the trade-off between residual energy of $\mathrm{CH}$ and the size of cluster. Heinzelman et al. [12] developed Low-Energy Adaptive Clustering Hierarchy (LEACH) protocol by combining energy-efficient clustering and medium access control (MAC). Qin et al. [13] proposed energy-efficient LEACH (EE-LEACH) and CMIMO where EE-LEACH performed network partitioning into fixed regions having equal angle that avoided the possibility of non-uniform $\mathrm{CH}$ distribution. In their approach, authors applied node location and the residual energy for $\mathrm{CH}$ selection. Singh.D et al. [14] proposed Enhanced Modified LEACH (EMODLEACH) protocol for WSN. Where the network can be classified into proactive, reactive and hybrid networks. Xiong et al. [15] applied network coding for an energy efficient CMIMO transmission and found that network coding reduces delay and energy consumption during inter-cluster communication. Singh.D et al. [16-17] proposed an improved energy routing protocol (IBRP) is an advanced version of LEACH protocol.in WSN. Fei et al. [18] exploited CMIMO and cluster based WSN to perform energy efficient communication. Authors found that the number of nodes involved in CMIMO based communication doesn't vary with the number of nodes having data for transmission. A distributed MIMO-adaptive energy-efficient clustering model was developed by Siam et al. [19] where authors applied multi-hop transmission. However, multi-hop retransmission caused energy exhaustion could not be addressed. Islam et al. [20] developed a channel condition aware $\mathrm{CH}$ selection and energy efficient CMIMO for WSNs. Cheng et al. [21] applied single-hop transmission and CMIMO to achieve energy efficient communication. 
Cui et al. [22] applied CMIMO by using multiple sensor nodes in the same cluster to perform cooperative communication. Authors stated that for inter-cluster communication there is no need of local information exchange where the nodes can communicate using Alamouti diversity codes. Gao et al. [23] developed a load-balanced cluster-based CMIMO transmission for WSN. Authors derived a two-layer hierarchy clustering model in which $\mathrm{CH}$ collects data using CMIMO communication. CMIMO was also used by Pillutla et al. [24] where they focused on the optimizing energy consumption and transmission rate. To achieve it authors developed a provably convergent block coordinate descent algorithm that estimates the rate and the number of clusters. Li et al. [25] explored CMIMO to perform inter-cluster and intra-cluster communication to preserve energy consumption. A similar effort was made by Chaibrassou et al. [26] where they applied a weighted link function to assist $\mathrm{CH}$ for cooperative nodes selection for data forwarding. They [27] developed a distributed Multi Channel CMIMO routing model for cluster based WSNs (MCMIMO) that at first organizes nodes into clusters and then the $\mathrm{CH}$ exploits weighted link function to identify the best cooperative nodes for data transmission. Nguyen et al. [28] applied Virtual MIMO (VMIMO) concept to derive a distributed Cooperative Clustering Protocol (CCP), where they used VMIMO diversity gain by performing $\mathrm{CN}$ selection within each cluster. Sharmila et al. [29] used the relationship between the data generated by each node and the distance between them to perform clustering. Applying CMIMO and data aggregation techniques authors derived energy efficient WSN routing protocol. Jaafar et al. [30] applied distributed space-time coding technique based multihop VMIMO for WSNs. Jayaweera et al. [31] applied multiplexing gain of VMIMO to obtain energy efficient and low overhead routing in WSN. Yuan et al. [32] applied cluster-based CMIMO to alleviate the impacts caused due to the radio irregularity and fading in multi-hop transmission. Malleswari et al. [33] and Mamun et al. [34] developed a cluster-based VMIMO for energy-constrained WSN communication. Authors applied Space-Time Block Coding (STBC) based VMIMO in conjunction with LEACH to achieve energy efficient routing. Sachan et al. [35] examined the impact of distance and long range distances on the selection of MIMO, MISO, SIMO, and SISO. Tiwari et al. [36] developed VMIMO for energy-efficient WSNs using an analytical approach. Wang et al. in [37] applied a clustered WSN in which nodes were distributed randomly within a circle area around single $\mathrm{CH}$ at the center. Once $\mathrm{CH}$ broadcasts source message to the neighboring nodes, those neighboring nodes which have successfully decoded the message were used to relay data towards sink using STBC to construct VMIMO. Ding et al. [38] developed a clusterbased VMIMO technique for energy-constrained WSN communication. Xu et al. [39] developed VMIMO coupled with multi-hop transmission, where a cross-layer model was derived using transmission rate, clusters and the virtual antenna nodes. Medhia et al. [40] applied mobility and CMIMO for WSN. To enable CMIMO communication each mobile node applied Alamouti diversity algorithm. The mobile sensor could move to a defined network location to collect sensed data and transmit it to the sink using CMIMO [41]. However, their approach lacked proactive node management that could have made it more realizable for real time system. Vidhya et al. [42] extended LEACH and used multi-hop transmissions to derive a cluster-based CMIMO for WSN. Cui et al. [43] examined CMIMO with Alamouti code for single-hop transmissions over WSN. Jayaweera et al. [44] examined CMIMO efficiency in terms of energy consumption and training overhead, where they found that CMIMO outperforms SISO-based WSNs. Sathian et al. [45] applied game theory to derive a $\mathrm{CH}$ Cooperative Trustworthy Energy Efficient MIMO (CH-C-TEEM) routing protocol. Islam et al. [46] developed cooperative communication approach, where the selected sensors at the transmitter form a MIMO. In their approach, the selection of nodes at the transmitter was done using the network parameters such as channel condition, residual energy; inter node distance and location. Zhang et al. [47] derived a cooperative node selection model for WSN under energyconstrained scenarios. Authors combined the residual energy and the link quality between the $\mathrm{CHs}$ to perform data transmission. Chattha [48] developed an energy balancing intelligent clustering model based MIMO which was applied in conjunction with the optimal forwarding/receiving node selection. Authors [49] derived a cluster size optimization model using the concept of the spatially correlation in data. Authors [50] focused on balancing the energy consumption between $\mathrm{CHs}$. The underlying principle behind the work in was that $\mathrm{CHs}$ near the sink require relaying more traffic than outsider $\mathrm{CHs}$ and therefore undergoes high energy depletion. Energy Based Clustering Self organizing map (EBCS) based clustering was developed by Enami et al. [51]. Amri et al. [52] developed a Multi-hop Hierarchical Routing Protocol using Fuzzy Logic (EMHR-FL), where Fuzzy Logic Inference System (FIS) was used to perform next-hop selection by considering residual energy of $\mathrm{CHs}$, distance between CHs and node density. Kubo et al. [53] performed multiple cooperative nodes selection by using "quality" and "angle" metrics.

\section{Problem formulation}

As discussed in previous section, a few efforts have been made to exploit the efficacy of enhanced clustering, better $\mathrm{CH}$ selection and CMIMO to enable energy efficient communication over WSNs. Most of the existing approaches either apply single parameter like residual energy, distance or link quality for $\mathrm{CH}$ selection. The reliability of such approaches often remains questionable, especially when a node undergoes mobility and hence dynamic topological conditions. On contrary, introducing mobile sink in clustering based WSN can enhance overall communication efficiency by avoiding multi-hop transmission that consumes significant energy during multi-node traversal and retransmission. Additionally, it can be vital for time efficient data gathering for QoS provision. In clustering based routing, the efficiency of clustering and $\mathrm{CH}$ selection often play vital role. Therefore, enhancing clustering and $\mathrm{CH}$ selection by exploiting key network variables such as internode distance, degree of dependence, etc can be significant. Furthermore, the use of multiple network parameters for $\mathrm{CH}$ selection can also be a revitalizing effort. In major existing system classical peer to peer transmission model is applied that reserves bandwidth for predefined node data transmission. It affects overall resource utilization efficiency of the routing protocol. Additionally, multi-hop transmission imposes significant retransmission and signaling overhead that leads energy exhaustion. To alleviate these issues CMIMO transmission can be a potential approach. In addition, the use of a single mobile sink to collect sensed data from $\mathrm{CHs}$ and $\mathrm{CNs}$ can also make overall routing 
protocol delay and energy efficient.

Considering large scale network, unlike classical clustering, in our routing protocol at first a large scale network is split into groups, which is then followed by clustering in each group. In Q-E2RPC routing protocol a hybrid clustering approach having dual phase implementation is developed, where in first phase FCM algorithm is applied that exploits inter-node distance to cluster nodes. In the second phase, an enhanced EM model is applied that exploits degree of dependence of a node on cluster to perform final clustering, before executing $\mathrm{CH}$ selection. This combined model ensures optimal number of clusters in the network that eventually reduces energy exhaustion and signaling overheads. Furthermore, considering significance of $\mathrm{CH}$ selection in WSN, the use of multiple network parameters can be vital. In $\mathrm{CH}$ selection model, node information, residual energy, Signal to Noise Ratio (SNR) or RSSI and node responsiveness are taken into consideration. The use of a single parameter such as the residual energy or the distance can be easy to decide $\mathrm{CH}$ of a cluster; however selecting $\mathrm{CH}$ with multiple parameters even under dynamic topology can be a trivial task. To deal with it, Q-E2RPLC incorporates FLC that learns multiple network states to perform optimal $\mathrm{CH}$ selection. In practice, multi-hop transmission intends to assure reliable transmission without data loss, however at the cost of retransmission and higher energy consumption. To alleviate such issues, introducing mobile sink can be vital, where a mobile sink can collect data from the $\mathrm{CHs}$ directly to avoid unwanted traversal and resulting energy exhaustion. To further strengthen the mobility model certain trajectory estimation approach such as travelling salesman problem etc can also be applied. Q-E2RPC protocol exploits CMIMO transmission features to perform inter-node communication from $\mathrm{CH}$ to mobile sink. This as a result makes overall communication energy, delay and resource efficient.

\section{Proposed System}

\subsection{Mobile-WSN Model}

Considering real-time communication where there can be a large number of sensors distributed across the field, QE2RPC has been developed with dense network with multiple sensor nodes. Such communication environment can be manufacturing establishments, schools/universities, hospitals, surveillance systems, border security etc. To alleviate the probability of contention and data losses during traversal across large network, Q-E2RPL protocol splits overall network into sub-network or regions called "Group". An illustration of the applied network region is given in Fig. 1 .

In Fig.1, circles presents $\mathrm{N}$ sensors distributed across the network with dimension $\mathrm{L} \times \mathrm{L}$. Here, the solid circle $\mathrm{C}$ presents the $\mathrm{CH}$ formed, which is supposed to be visited by the mobile sink for data collection by means of CMIMO transmission scheme. The solid-fill circles refer nodes in a group, while dotted region presents the cluster. Here, the term "Group" signifies a set of nodes which are able to communicate with each other. The nodes in other groups are unable to communicate due to high inter-node distance. Let $\mathrm{G}$ be the total number of groups in the network and $\mathrm{N}_{\mathrm{g}}$ and $\mathrm{C}_{\mathrm{g}}$ be the total number of sensor nodes and the number of clusters in gth group, respectively. Unlike traditional approaches, the total number of groups is estimated by exploiting the location of the nodes and its radio range $\mathrm{R}$.
Here, each $\mathrm{CN}$ shares its information to perform $\mathrm{CH}$ selection. Once receiving the data from $\mathrm{CNs}, \mathrm{CH}$ forwards it to the mobile sink using CMIMO transmission. In Q-E2RPC, each $\mathrm{CH}$ operates as the cooperative transmitter, receiving the data packet processes for encoding by means of algorithms STBC. It is then followed by the transmission to the mobile sink. In cooperative communication, the receiving CHs can use Channel State Information (CSI) to decode the STBC data and the decoded data can be relayed to the sink for further process.

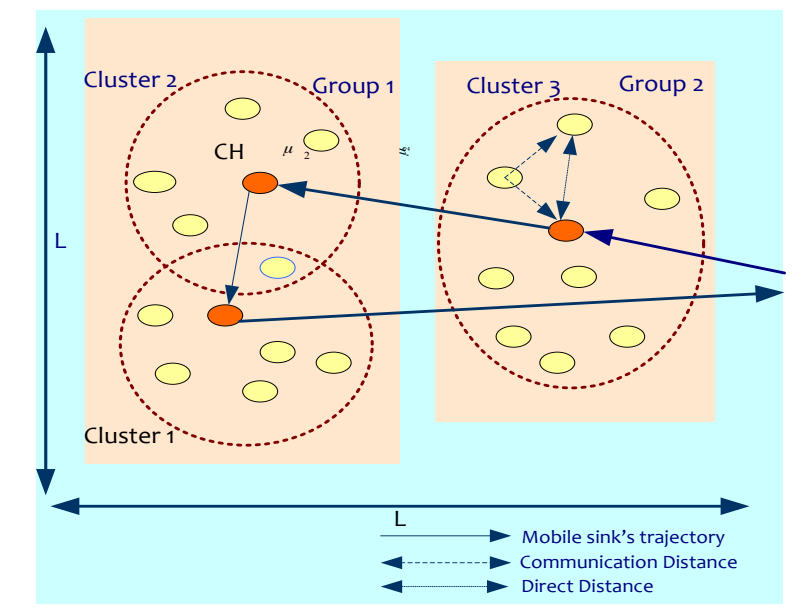

Fig.1. Mobile-WSN Network

In Q-E2RPC, it is assumed that each node is aware of its own position and cluster's location. Practically, it can be achieved through certain node positioning algorithm. Here, each node has a definite communication range $\mathrm{R}$ and therefore transmission can be successful only within R. Each node possesses a definite amount of memory to store sensed data to forward it to the sink. CHs collect sensed data from $\mathrm{CNs}$ through CMIMO transmission approach and meanwhile mobile sink collect data from $\mathrm{CHs}$ in the similar manner. To avoid unwanted multi-hop traversal, in Q-E2RPC a single mobile sink has been applied that assures timely data gathering to enable swift decision. It not only reduces traversal time but also reduces energy exhaustion due to (multi-hop) retransmission.

The efficiency of a clustering based WSN routing protocols depends on two key factors, clustering and $\mathrm{CH}$ selection. The use of real-time network parameters such as network link, radio strength, energy level and channel gain etc can make $\mathrm{CH}$ selection more efficient. Such hypotheses have been asserted by researcher [54] where authors applied RSSI, energy level, channel gain and distance to perform $\mathrm{CH}$ selection. However, applying multiple parameters to decide $\mathrm{CH}$ is intricate and can become more trivial under dynamic topology. To alleviate such issues, in our model FLC algorithm has been applied that learns the network parameters to perform $\mathrm{CH}$ selection. Here, it should be noted that in Q-E2RPC, before executing clustering and $\mathrm{CH}$ selection, we have executed network partitioning that splits overall network into two groups that makes overall computation efficient. Each node forwards sensed data to the connected $\mathrm{CH}$ via CMIMO transmission where $\mathrm{CH}$ further combines those data to transmit towards the destination node. 4.2.System Design Q-E2RPC exploits the efficacy of the different enhancements made for clustering, $\mathrm{CH}$ selection, CMIMO transmission and mobile sink based data gathering to enable 
delay resilient and QoS communication over WSN. QE2RPC incorporates the following steps:

\section{Step-1 Fuzzy cluster mean (FCM) and Expectation}

Maximization based clustering,

Step-2 Fuzzy Logic Control (FLC) based CH selection,

Step-3 Cooperative communication based data transmission using Single Mobile Sink node.

A brief of the implementation model is given as follows:

\section{i. Dual Phase Clustering}

Unlike classical clustering approaches, we have applied dual phase clustering, where at first FCM is executed to perform initial clustering over two groups. Once performing initial clustering, we have executed EM based centralized clustering that uses the cluster information and associated CNs information to re-structure the clusters. A brief of this clustering paradigm is given as follows:

\section{FCM Based Initial Clustering}

Once performing grouping or the network partitioning, FCM [55] is executed that exploits inter-node distances to perform initial clustering. Typically, FCM is used to perform pattern recognition by providing membership to each data point related to each cluster center, where the summation of membership for all data points should be equal to one. In FCM based clustering we intend to minimize an objective function given in (1).

$$
\min _{\mu_{i j}, m_{j}}\left(v_{q}\right)
$$

Where

$v_{q}=\sum_{i=1}^{N} \sum_{j=1}^{M} \mu_{i j}^{q}\left\|\theta_{i}-m_{j}\right\|^{2}$,

Noticeably, the deployed WSN network considers node distribution in such manner that it depicts like a network graph $\mathrm{G}$, with each vertex signifying the node's position in 2D space. Mathematically, sensor node is positioned as $q_{i}=\left[X_{i}, Y_{i}\right]^{T}$ for the $i$ th node. Thus, in above equation, $q_{i}$ signifies the location parameter of a node. If the two nodes $i$ and $j$ are located within their communication or radio ranger, their link could be defined in terms of an edge. In (2) $M$ states the total number of clusters, q presents the fuzziness exponent $(>1), \mu_{i j}$ presents the degree of membership of ith node in $j$ th cluster and $m_{j}$ states the center of cluster $j$ and $m_{k}$ refers center of cluster $\mathrm{k}$. The value of $\mu_{\mathrm{ij}}$ exists in the range of 0 to 1 for each $\mathrm{CN}$ to the $\mathrm{CH}$. Here, we have performed fuzzy partitioning by means of an iterative optimization scheme where it intends to minimize the objective function given in $\mu_{\mathrm{ij}}(3)$.

$\mu_{i j}=\frac{1}{\sum_{h=1}^{M}\left(\frac{\left\|\theta_{i}-m_{j}\right\|}{\left\|\theta_{i}-m_{k}\right\|}\right)^{\frac{2}{q-1}}}$,

(3)

Finally the cluster center $m_{j}$ obtained is (4).

$m_{j}=\frac{\sum_{i=1}^{N} \mu_{i j}^{q} \cdot \theta_{i}}{\sum_{i=1}^{N} \mu_{i j}^{q}}$

Once reaching stopping criteria the iterative optimization is stopped. Noticeably, in FCM based clustering the stopping criteria is defined as

$\left\{\mu_{\mathrm{ij}}^{\mathrm{k}+1}-\mu_{\mathrm{ij}}^{\mathrm{k}}\right\}<\sigma$,

Where, $\mathrm{k}$ signifies the iteration step.

The snippet of the applied FCM based clustering is given as follows (Fig. 2):

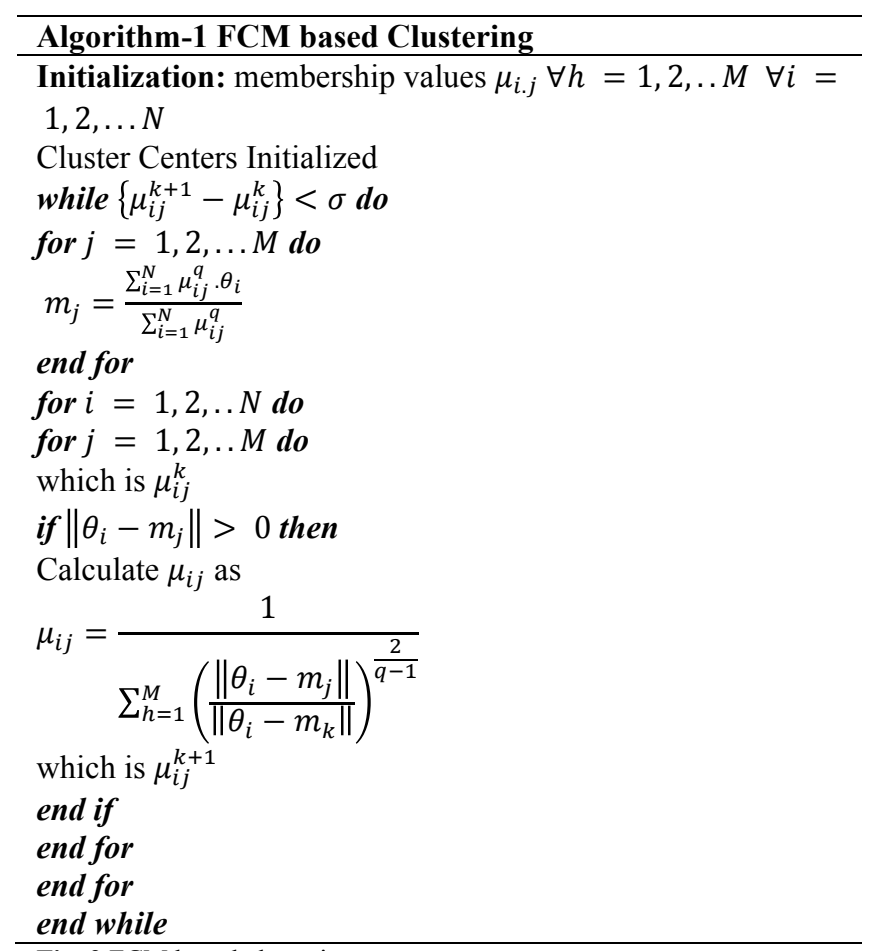

Fig. 2 FCM based clustering

To further strengthen clustering, a centralized clustering approach named EM has been applied.

\section{Expectation Maximization Based Clustering}

EM is a generic clustering model that assumes that all sensor nodes are distributed as per Gaussian Mixture Model (GMM) (5).

$E(\mathrm{x})=\sum_{c=1}^{C} \pi_{c} A\left(x \mid \sigma_{c}, \Sigma_{c}\right)$

In (5), variables $C$ and $\sigma_{c}$ present total clusters and a combination factor for the cth cluster, respectively. Here, $\mathrm{A}(\mathrm{x} \mid \sigma, \Sigma)$ is obtained using (6).

$A(x \mid \sigma, \Sigma)=\frac{1}{(2 \pi)|\Sigma|^{1 / 2}} \exp \left\{-\frac{1}{2}(x-\sigma)^{T} \Sigma^{-1}(x-\sigma)\right\}$,

In (6), $\mathrm{x}$ and $\sigma$ signify the location vector of CNs and the location vector of $\mathrm{CH}$ of the cth cluster, respectively. The variable $\Sigma_{\mathrm{c}}$ states a $2 \times 2$ Covariance Matrix $(\mathrm{CM})$ of the cth cluster. Unlike FCM, EM calculates Degree of Dependence (DoD) of each $\mathrm{CN}$ that is nothing else but the Responsiveness of a node on the connected cluster. We have estimated "Responsiveness" of a node $\mathrm{n}$ on kth cluster using (7).

$\varphi_{n c}=\frac{\pi_{c} A\left(x_{n} \mid \sigma_{C}, \Sigma_{C}\right)}{\sum_{j=1}^{c} \sigma_{j} A\left(x_{n} \mid \sigma_{j}, \Sigma_{j}\right)}$.

Typically, the value of responsiveness (7) remains in the range of 0 and 1 .

Once performing FCM based initial clustering and 
Kummathi Chenna Reddy, Geetha D Devanagavi and Thippeswamy MN/

Journal of Engineering Science and Technology Review 12 (1) (2019) 213 - 224

retrieving the location vector of the $\mathrm{CHs}$, the communication distances $\mathrm{D}_{\mathrm{nc}}$ between each $\mathrm{CN}$ and associated $\mathrm{CH}$ is estimated. In this way, two components location vector $(\sigma)$ and covariance matrix $(\Sigma)$ are obtained. Now, to deal with the complexity due to the large size (i.e., dense) network, the overall network is partitioned into groups. Once initiating the EEM based clustering phase, the proposed Q-E2PRC model selects a particular group with the highest value of the proportion of the number of residing nodes to the total cluster counts in group $g$. In other words Q-E2PRC routing protocol selects a group with the highest value of the parameter $v_{g}$, which is mathematically obtained by (8).

$v_{g}=\frac{C_{g}}{N_{g}}$

In (8), $C_{g}$ states the number of clusters in the group and $N_{g}$ states the total number of nodes in the group. Thus, in the selected group having the highest value of $v_{g}$, Q-E2PRC model selects all those nodes which belong to the group $g$ and updates the node responsibility factor, $\varphi_{n c}$ for these all nodes. In our proposed routing model the value of $\varphi_{n c}$ signifies the extent to which a node $n$ belongs to the cluster $k$. Thus, employing the updated responsibility factor, $\varphi_{n c}$ the cluster centroids, and covariance matrix are reestimated and the total number of nodes belonging to the $k$ th cluster is obtained using (9).

$N_{C}=\sum_{x_{n} \in X} \varphi_{n c}$

Thus, this process continues till the difference between newly estimated log-likelihood and the previously estimated $\log$ likelihood becomes lower than the value of $\epsilon$.

In proposed EM based CCP model, $\mathrm{C}$ signifying weighted Center of Gravity (CoG) of a 2D-location vector is estimated for each node. To achieve it, the responsiveness of each node (assuming it to be the weight of the node) is considered. Later, location of the $\mathrm{CH}$ can also be changed by the weighted CoG. In our model EM estimates the log likelihood to estimate optimal number of clusters using (10).

$\mathbb{L}=\ln E(X \mid \sigma, \Sigma, \sigma)=\sum_{n=1}^{N} \ln \left\{\sum_{c=1}^{C} \sigma_{c} A\left(x_{n} \mid \sigma_{c}, \Sigma_{c}\right)\right\}$

Similar to the FCM based approach; EM continues iterating until convergence. The value of (10) reduces uneventfully thus making EM terminate. EM updates the key information such as, $\sigma_{\mathrm{c}}$ and $\varphi_{\mathrm{nc}}$ of each node to the cth cluster that eventually leads reduction in the Sum of Square (SoS) of the distance between each node and cluster. It finally gives the optimal clustering results. Once performing clustering, $\mathrm{CH}$ selection is performed for each cluster for which FLC is applied that learns over the network parameters to select the best node so as to become the $\mathrm{CH}$ for further data transmission.

\section{ii. Cluster Head (CH) Selection}

The selection of $\mathrm{CH}$ often plays decisive role in assuring optimal performance. With this motivation, we have applied multiple network parameters to perform $\mathrm{CH}$ selection. The parameters being used in our model are as follows:

- Location information of each sensor node within the cluster,

- Distance of each sensor node with respect to the base station of sink location,
- Residual energy of each node, and

- SNR of the reporting channel of the $\mathrm{CH}$ and base station.

Here, location information enables the selection of $\mathrm{CH}$ near CNs that consequently minimizes multi-hop transmission and hence energy consumption. The distance between CNs, $\mathrm{CHs}$ and mobile sink can affect the transmission efficiency, even with CMIMO. Hence, we perform $\mathrm{CH}$ selection in such manner that the distance between $\mathrm{CNs}$ and $\mathrm{CH}$ is lower enough to communicate in single-hop. Since, $\mathrm{CH}$ is selected near $\mathrm{CNs}$ and hence, the distance between $\mathrm{CH}$ and mobile sink might increase. Under such scenarios, the probability of link failure can't be ignored. Considering such issues, we have used SNR of the link from $\mathrm{CH}$ to the sink as an additional parameter for $\mathrm{CH}$ selection. Realizing the fact that the successful data transmission also depends on the residual energy of the forwarding node, we have used residual energy of each node to assess its suitability to become the $\mathrm{CH}$ of that cluster. Exploiting multiple parameters for $\mathrm{CH}$ selection can be an intricate task that becomes more tedious during topological variations (due to sink mobility). To deal with this, we have applied FLC that learns over the network parameters to decide $\mathrm{CH}$ for each cluster. In our model, we have applied four key network parameters including distance (i.e., node position), residual energy, SNR and responsiveness to decide optimal $\mathrm{CH}$ for a cluster. The decision variables and respective conditions are presented in Table 2.

Table 2. CH Selection Conditions

\begin{tabular}{c|c}
\hline Parameters & Suitability \\
\hline Distance & Low \\
Residual Energy & High \\
SNR & High \\
Responsiveness & High \\
\hline
\end{tabular}

FLC model learns over the network parameters and intends to achieve a defined objective function. To select $\mathrm{CH}$ of $\mathrm{mth}$ cluster, an exclusive objective function is derived (11).

$\Psi_{i}^{(m)}=\max _{C H}\left(\frac{\mho_{i}^{(m)} \gamma_{i}^{(m)} \varphi_{i}^{(m)}}{\alpha P L_{i}^{(m)}+(1-\alpha) P L_{M S}^{(m)}}\right)$

Where $\mho_{i}^{(m)}$ presents the residual energy of ith sensor (i.e., $\mathrm{CN}), \quad \gamma_{\mathrm{i}}^{(\mathrm{m})}$ signifies the SNR of the link between the ith node and the sink, and $\varphi_{\mathrm{i}}^{(\mathrm{m})}$ refers the responsiveness of ith node. The other parameter $\mathrm{PL}_{\mathrm{i}}^{(\mathrm{m})}$ presents the average path loss of the channels between the ith node and the other CNs. The variable $\mathrm{PL}_{\mathrm{MS}}^{(\mathrm{m})}$ refers path loss of ith $\mathrm{CN}$ and sink, and $\alpha$ presents a weight parameter assigned to the path loss components $\mathrm{PL}_{\mathrm{i}}^{(\mathrm{m})}$ and $\mathrm{PL}_{\mathrm{MS}}^{(\mathrm{m})}$, i.e., $\mathrm{CN}$ to $\mathrm{CH}$ and $\mathrm{CH}$ to the mobile sink, respectively. In (11) the value of $\alpha$ exists in between 0 and 1 . The distances between nodes are also maintained low between possible $\mathrm{CH}$ and $\mathrm{CNs}$. We have estimated the path loss $\mathrm{PL}_{\mathrm{i}}^{(\mathrm{m})}$ using (12).

$P L_{i}^{(m)}=\frac{\sum_{i=1}^{N m} \mathrm{PL}_{\mathrm{i}, \mathrm{j}}^{(\mathrm{m})}}{N_{m}}$

Where $\mathrm{N}_{\mathrm{m}}$ presents the total number of sensor nodes in the mth cluster. The path loss between the ith sensor and CNs (i.e., ith sensor) is given by $\mathrm{PL}_{\mathrm{i}, \mathrm{j}}^{(\mathrm{m})}$. Mathematically,

$P L_{i, j}^{(m)}=10 n \log _{10}\left(R_{i, j}^{(m)}\right)$ 
In $(13),\left(\mathrm{R}_{\mathrm{i}, \mathrm{j}}^{(\mathrm{m})}\right)=\left\|\theta_{\mathrm{j}}^{(\mathrm{m})}-\theta_{\mathrm{i}}^{(\mathrm{m})}\right\|$ gives the distance between jth $\mathrm{CN}$ and ith $\mathrm{CH}$, where the position of the ith $\mathrm{CH}$ is $\theta_{\mathrm{i}}^{(\mathrm{m})}=\left\{\mathrm{x}_{\mathrm{i}}^{(\mathrm{m})}, \mathrm{y}_{\mathrm{i}}^{(\mathrm{m})}\right\}$ and the position of the $\mathrm{jth}$ node is $\theta_{j}^{(m)}=\left\{x_{j}^{(m)}, y_{j}^{(m)}\right\}$. The variable $n$ presents the path loss exponent. The path loss between $\mathrm{CH}$ of $\mathrm{mth}$ cluster and sink is obtained as (14)

$P L_{M S}^{(m)}=10 n \log _{10}\left(R_{M S}^{(m)}\right)$

Where $\mathrm{R}_{\mathrm{MS}}^{(\mathrm{m})}=\left\|\theta_{\mathrm{i}}^{(\mathrm{m})}-\theta_{\mathrm{MS}}\right\|$, with $\theta_{\mathrm{MS}}=\left\{\mathrm{x}_{\mathrm{ms}}, \mathrm{y}_{\mathrm{ms}}\right\}$ as the mobile sink location. Estimating network parameter, FLC is executed that performs $\mathrm{CH}$ selection. The $\mathrm{CH}$ selection algorithm is given as follows:

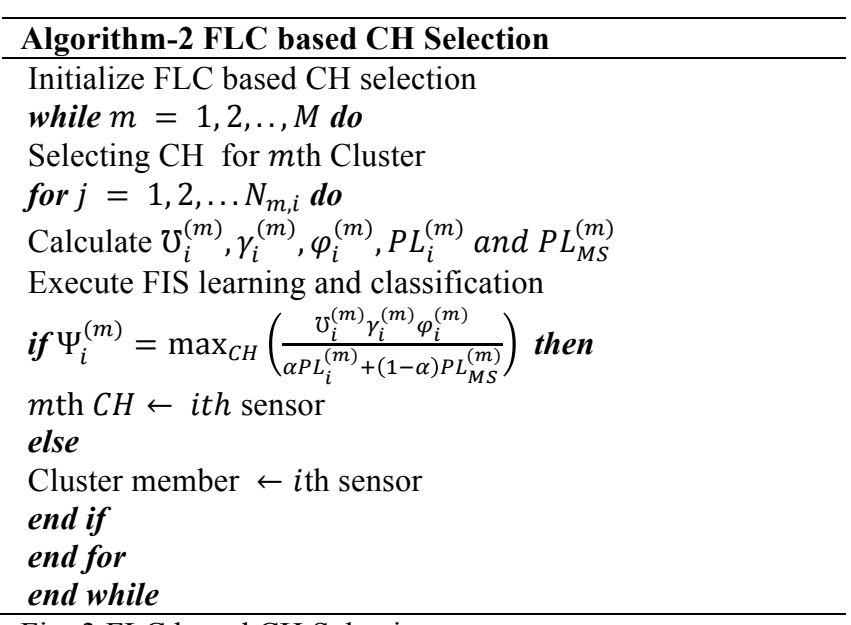

Fig. 3 FLC based CH Selection

\section{iii. CMIMO Based Data Transmission}

Once performing clustering and $\mathrm{CH}$ selection, the mobile sink starts patrolling to collect data from CHs. Here, it should be noted that the mobile sink collects data from $\mathrm{CHs}$ where the sensor data are collected by exploiting cooperative MIMO paradigm. The use of a single mobile sink might lead a situation where it could not be able to reach $\mathrm{CHs}$ for data collection. In such cases the probabilities of data loss and delay can't be ignored. Additionally, as the speed of mobile node is comparatively slower than the electrical communication, enhancing movement pattern of the mobile sink remains an open challenge. To deal with this problem, heuristic approaches such as Traveling Salesman Problem (TSP) can be applied to assist sink to reach $\mathrm{CHs}$ for early data gathering. To collect data from $\mathrm{CHs}$, we have applied MIMO technique. In Q-E2RPC protocol $\mathrm{CHs}$ transmit data to the mobile sink using MIMO, where to collect data; mobile sink transmits a Data Transmission Request (DTR) to the $\mathrm{CHs}$ that further broadcasts to the CNs. Receiving DTR response from $\mathrm{CH}$, mobile sink re-sends the data transmission request which is followed by relaying data from $\mathrm{CH}$ to the mobile sink. One more novelty introduced in Q-E2RPC is the provision of direct communication between $\mathrm{CNs}$ and mobile sink. Typically, CNs transmit data to the connected $\mathrm{CH}$, that then forwards it to the mobile sink; however the in-depth analysis shows the scope for further enhancement, particularly for delay efficient transmission. In Q-E2RPC, transmission is scheduled in such manner that finding mobile sink nearer than the $\mathrm{CH}$, a $\mathrm{CN}$ can transmit sensed data to the mobile sink directly that avoids transmission delay caused during transmission through $\mathrm{CH}$. To minimize energy consumption during transmission, here
CNs transmits data based on the responsiveness factor. As discussed, responsiveness is estimated using parameters $\mu, \sigma$, and $\Sigma$, and hence these parameters can be appended to the DTR message to be transmitted back to the mobile sink. Once deploying CNs in the network, each node exchanges its location information with all $\mathrm{CNs}$ in the same cluster. Since, this exchange is performed only once after sensor nodes deployment that reduces the unwanted signaling overhead. In case a node belongs to multiple clusters, it can use responsiveness factor to decide best $\mathrm{CH}$ to forward data. For example, in case a node has the responsiveness of $\varphi_{\mathrm{n} 1}=$ 0.8 and $\varphi_{\mathrm{n} 2}=0.2$. Once receiving DTR from $\mathrm{CH}$ of the cluster 1 , nth node transmits $80 \%$ of data. In case it receives DTR from cluster 2 , it transmits remaining $20 \%$ of data to the mobile sink through $\mathrm{CH}$ of the second cluster. It ensures that the data reaches to the sink reliably and timely to meet QoS demands.

\section{Signaling Overhead Optimization}

In Q-E2RPC, once reaching $\mathrm{CH}$ of a cluster, mobile sink transmits DTR message to invite data transmission from $\mathrm{CH}$ and the associated CNs. Once receiving DTR message, CNs transmits its sensor data to the $\mathrm{CH}$ that later forwards it to the mobile sink. CNs then broadcast DTR to the neighboring sensor nodes and it continues till $\mathrm{CH}$ has received it. Here, the possibility of getting DTR twice or multiple-times can't be ignored that might increase signaling overhead significantly. $\mathrm{CN}$ at first transmits its data and then broadcasts DTR. However, the uncontrolled broadcast might cause signaling overhead imposing excessive energy exhaustion, mainly due to unwanted redundant packet transmission. Under such conditions, reducing redundant packet transmission and DTR are must. Fig. 4 illustrates DTR caused message flooding, where it can be observed that the higher connectivity may require higher DTR messages and hence can led signaling message flooding.

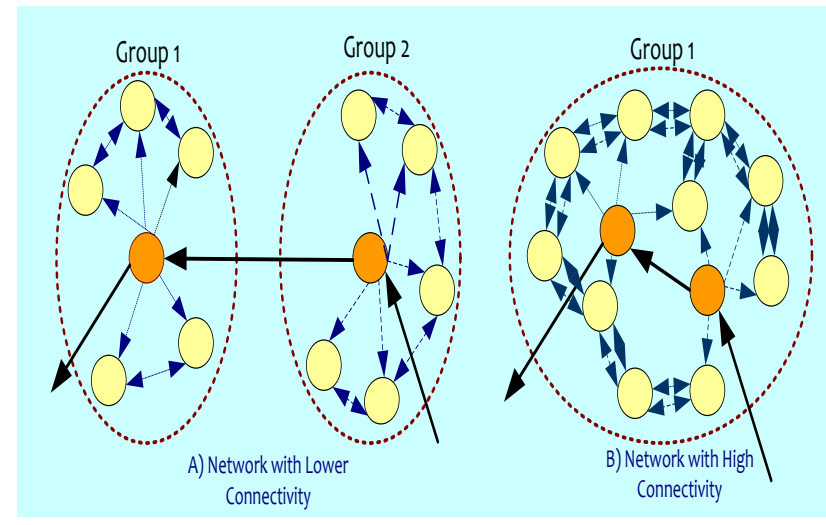

Fig. 4 Illustration of the DTR Signaling flood with different connectivity scenario

Fig. 4 illustrates WSN with two groups containing a total of 12 nodes. In the network with two CHs, the mobile sink requires traversing twice to transmit DTR to the connected CNs. It also presents that the nodes in a group can communicate only with the CNs of that group (Group-1). Mobile sink transmits DTR to each $\mathrm{CN}$ in cluster 1, and then each $\mathrm{CN}$ broadcasts transmission request. It illustrates a scenario where CNs can communicate to each other and broadcasts DTR. In this case when each node transmits DTR the total number of messages becomes higher. Though, the number of sensor nodes and $\mathrm{CHs}$ remains the same, the number of DTR increases with increase in connectivity. It 
Kummathi Chenna Reddy, Geetha D Devanagavi and Thippeswamy MN/

Journal of Engineering Science and Technology Review 12 (1) (2019) 213 - 224

depicts that the total number of DTR increases as per increase in the number of clusters. To reduce DTR maintaining optimal number of clusters can play a vital role. The FCM and EM based clustering assisted our model to achieve QoS oriented, energy and delay efficient communication over WSNs.

\section{Results and Discussion}

In this research the overall emphasis was made on developing a novel QoS oriented and energy efficient WSN routing protocol, Q-E2RPC by employing enhanced clustering approach, $\mathrm{CH}$ selection, cooperative communication and single mobile sink based data collection. Considering the large scale network size, at first we performed network partitioning that structured overall network into two groups. Now, realizing the significance of the optimal number of clusters in the network, a two phase clustering model was developed. In our proposed clustering method at first FCM was applied to perform initial cluster formation, which was then followed by EM based clustering. In Q-E2RPC, EM based clustering exploited initial cluster information to perform clustering, which was then followed by the use of multiple network parameters like inter-node distance, residual energy of node, signal to noise ratio and responsiveness of the node in a cluster to perform final clustering. The derived network parameter called Responsiveness factor played vital role in enabling efficient clustering that directly affects number of $\mathrm{CHs}$ across network. Considering multiple decision variables, we applied FLC that estimated best node as $\mathrm{CH}$ of each clusters. Unlike classical methods of data transmission, Q-E2RPC incorporated cooperative communication amongst $\mathrm{CNs}$ and associated $\mathrm{CHs}$, and then $\mathrm{CHs}$ to the mobile sink. To alleviate the issue of excessive energy consumption (due to multi-hop MIMO transmission); we deployed a mobile sink to collect data from each $\mathrm{CH}$ for QoS delivery and energy efficient communication. Noticeably, in Q-E2RPC the mobile sink movement was controlled based on data request from CHs. The routing model was developed in way that it not only focuses on energy efficiency but also assures QoS delivery by maintaining minimum end-to-end delay, bandwidth utilization and higher reliability. The use of CMIMO helped Q-E2RPC to perform communication between $\mathrm{CNs}$ and respective $\mathrm{CHs}$ and $\mathrm{CH}$ to the mobile sink. The enhancement caused due to efficient clustering, $\mathrm{CH}$ selection strengthened timely data delivery, energy efficiency as well as significant reduction in computational complexity or signaling overheads. Q-E2RPC is developed over IEEE 802.15.4 MAC. The total simulation time is 800 seconds, where the transmission was tested with $2 \mathrm{~kb} / \mathrm{sec}$. To assess performance of Q-E2RPC protocol, we have developed simulation model using Network Simulator tool commonly known as NS2. Further, to plot the graphs MATLAB 2015a tool was used. The simulation environment considered in this study is given in Table III.

We deployed multiple sensor nodes randomly, where a single mobile node was also deployed as sink (Node in Green color in Fig. 6). In Q-E2RPC (FCM and EM based clustering) to enable swift and efficient communication over WSN, we performed network partitioning. In Q-E2RPC, we partitioned overall network into two groups (Fig. 6). Noticeably, the connected sensor nodes of one group can communicate only with the nodes connected to it. In other words, the nodes connected to one group can't communicate with the nodes of other group (Fig. 6).

Table 3. Simulation Environment

\begin{tabular}{l|l}
\multicolumn{1}{c|}{ Parameter } & \multicolumn{1}{c}{ Value } \\
\hline MAC & IEEE 802.15 .4 \\
Efficiency of RF power amplifier & 0.47 \\
Link margin & $40 \mathrm{~dB}$ \\
Gain factor & $30 \mathrm{~dB}$ \\
Power density of AWGN channel & $-134 \mathrm{dBm} / \mathrm{Hz}$ \\
Noise Figure (Receiver) & $10 \mathrm{~dB}$ \\
Path loss & $3-5$ \\
Carrier frequency & $2.5 \mathrm{GHz}$ \\
Bandwidth & $20 \mathrm{KHz}$ \\
BER performance & $10-3$ \\
Transmitter circuit power consumption & $98.2 \mathrm{mw}$ \\
Receiver circuit power consumption & $112.6 \mathrm{mw}$ \\
Antenna gain of Transceiver & $5 \mathrm{~dB}$ \\
Routing table update (exchange) & 5 \\
period for each round & \\
Routing table size & 100 \\
Transmission rate & $2 \mathrm{p} / \mathrm{sec}$ \\
Packet size & $2 \mathrm{kbits}$ \\
Transmission probability of each node & 0.8 \\
\hline
\end{tabular}

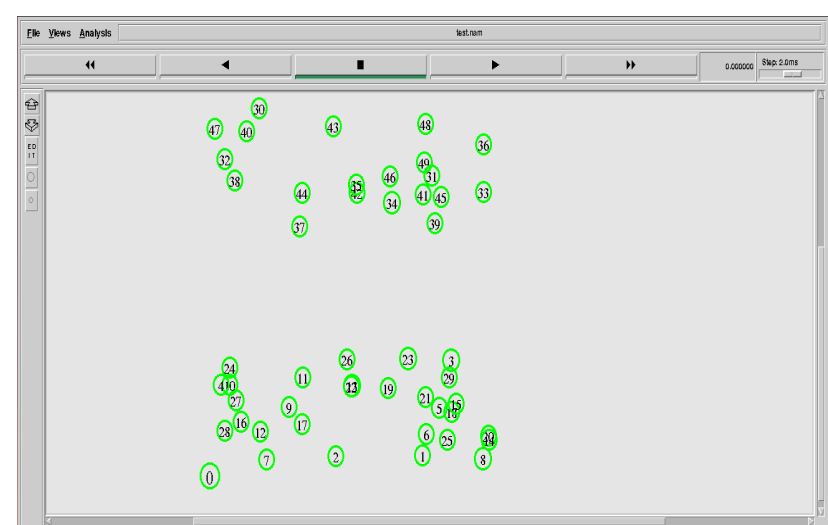

Fig. 5 Random node distribution across the WSN network

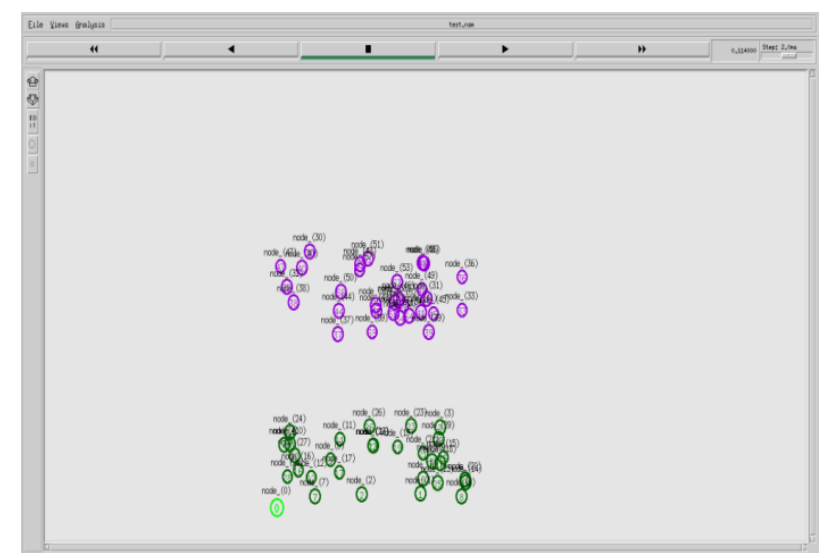

Fig. 6 Network partitioning into two groups

Once the nodes were partitioned into two groups, we executed clustering in which at first we applied FCM clustering that exploited inter-node distance to obtain initial clusters. However, realizing the need to incorporate node responsiveness to enhance clustering, we applied EM based clustering. Thus, based on the above discussed dual phase clustering we obtained the optimal number of clusters as depicted in Fig. 7. In Fig. 7, different clusters with different colors are presented. Once performing clustering, the node information including node position, signal to noise ratio, 
Kummathi Chenna Reddy, Geetha D Devanagavi and Thippeswamy MN/

Journal of Engineering Science and Technology Review 12 (1) (2019) 213 - 224

residual energy of each node and responsiveness of a node to the belonging cluster was used to perform $\mathrm{CH}$ selection. The $\mathrm{CH}$ estimated for each cluster can be visualized in Fig. 8 (black square box).

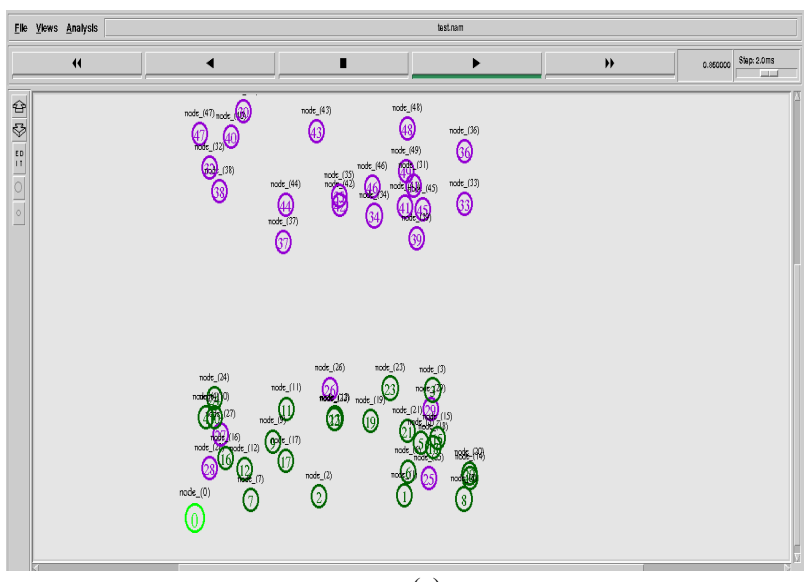

(a)

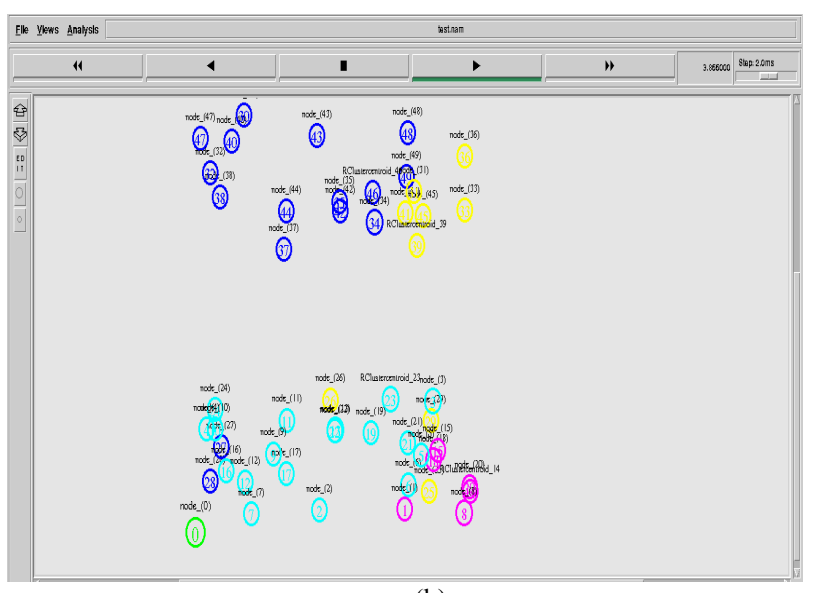

(b)

Fig. 7 (a).FCM assisted Initial Clustering, (b) .FCM+EM based clustering for Q-E2RPC routing protocol.Dual phase clustering results

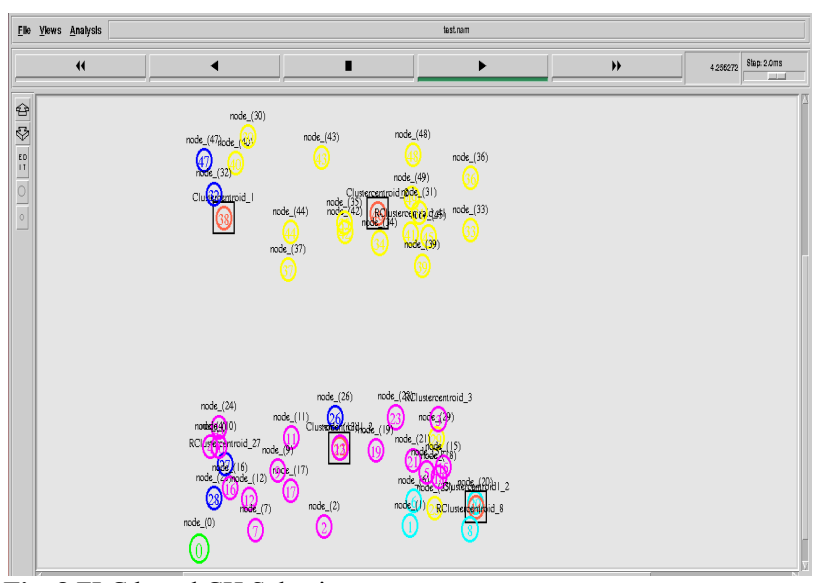

Fig. 8 FLC based CH Selection

Once performing $\mathrm{CH}$ selection, CMIMO transmission was applied to perform communication between $\mathrm{CN}$ and connected $\mathrm{CH}$, which is later transmitted to the mobile sink for further decision process. A snippet of cooperative communication between $\mathrm{CHs}$ and mobile sink is depicted in Fig. 9.

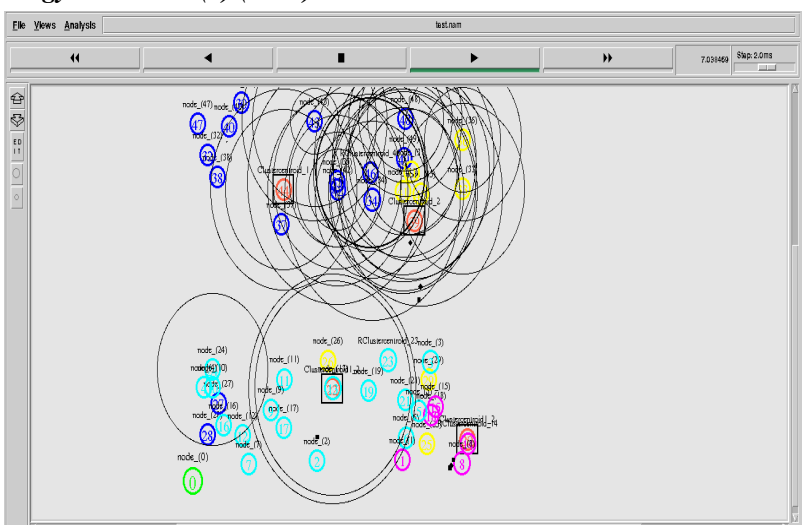

Fig. 9 Cooperative MIMO based data transmission (Mobile sink Location-1)

In Fig. 9, the initial location of mobile sink (green color) can be visualized that after certain time moves to the other location to collect data from CHs (Fig. 10). Noticeably, in Q-E2RPC nodes communicate in cooperative MIMO approach.

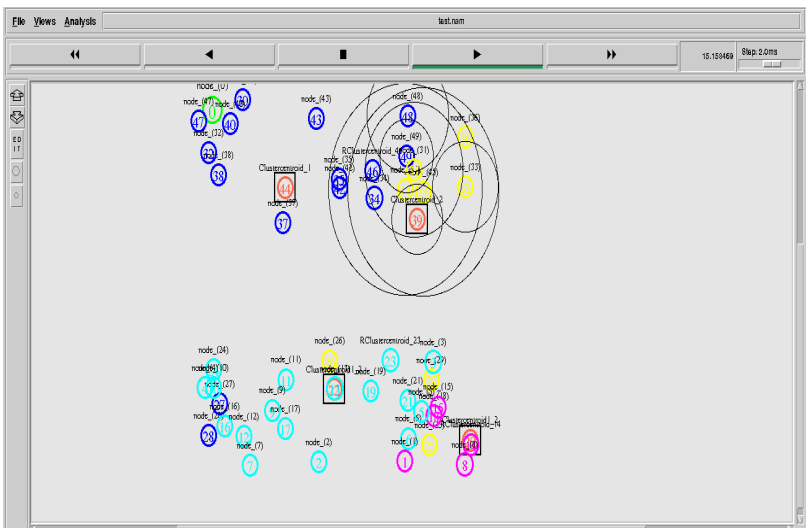

Fig. 10 Cooperative MIMO based data transmission (Mobile sink Location-2)

Noticeably, in Fig. 9 and Fig. 10, circles do signify data transmission and respective radio range. The large circles represent the radio range of the $\mathrm{CH}$ that reveals that the radio range of $\mathrm{CH}$ is sufficient enough to maintain communication with $\mathrm{CNs}$ belonging to that cluster.

To assess the relative performance of Q-E2RPC, we simulated FCM based clustering at first, which has been followed by FCM and EM as cumulative clustering approach. Thus, the respective performance for FCM and $\mathrm{FCM}+\mathrm{EM}$ (this is the clustering model applied in our proposed Q-E2RPC routing protocol) was obtained. For $\mathrm{CH}$ selection we have applied FLC. The results obtained in terms of packet delivery ratio (PDR), delay, energy consumption and efficiency are given in Fig. 11, Fig. 12, Fig. 13 and Fig. 14, respectively. Observing overall results it can be found that the proposed Q-E2RPC routing protocol enables better clustering thus augmenting overall performance. Q-E2RPC performs better than FCM based clustering. Here, the effectiveness of responsiveness clustering model can be visualized. This is because responsiveness assesses connectivity and degree of dependence of a node with its cluster that assures that a node having higher connectivity with a cluster would yield more efficient communication. Mobile sink node has strengthened Q-E2RPC to exhibit delay efficient communication. Nondeniably, CMIMO based communication among CNs and $\mathrm{CH}$ and further between $\mathrm{CHs}$ to mobile sink has further 
Kummathi Chenna Reddy, Geetha D Devanagavi and Thippeswamy MN/

Journal of Engineering Science and Technology Review 12 (1) (2019) 213 - 224

strengthened energy efficiency and higher throughput. As depicted in the results (Fig. 11 to Fig. 14), FCM refers simulation outcome with only FCM based clustering. In addition to it, we have developed a multi-hop transmission by exploiting K-Conid [56] based clustering, which comes under the category of centralized clustering method. Here, it should be noted that in all these simulations, we have applied FLC based $\mathrm{CH}$ selection and MIMO based transmission.

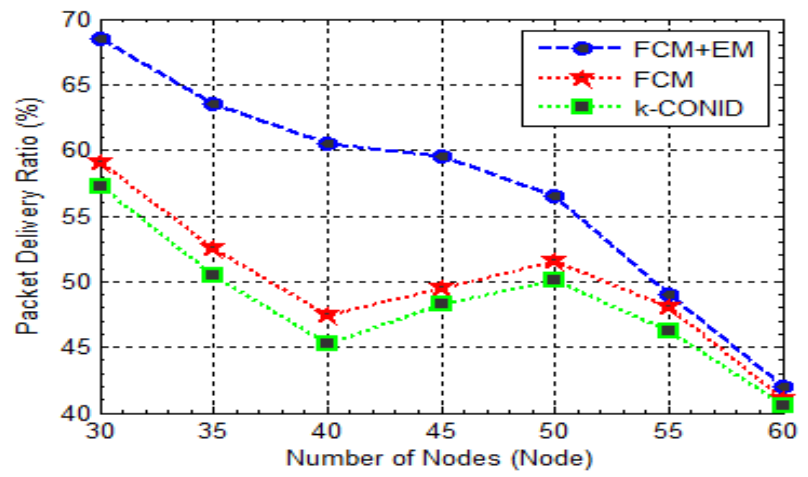

Fig. 11 Packet delivery ratio

Observing results, it can be found that Q-E2RPC outperforms both FCM and K-Conid clustering based routing. Fig. 11 presents the comparison of PDR between QE2RPC and FCM. The efficiency of FCM and EM for clustering optimization (i.e., maintaining optimal number of cluster to avoid energy consumption while assuring higher throughput and connectivity) can be visualized in Fig. 11. Fig. 12 shows delay of all three simulation scenarios, where Q-E2RPC has shown minimum delay. In achieving such augmented results role of mobile sink can't ignored. Its impact can be seen in Fig. 13 and Fig. 14 . Considering residual energy as one of the key decisive factor for success MIMO transmission, we estimated the energy consumed, $\mathrm{E}_{\text {Trans }}$ during transmission from sensor nodes to the mobile sink. Here, $\mathrm{E}_{\text {Trans }}$ states the energy required to transmit data from the sensor nodes to the mobile sink. If a sensor node is far away from its associated $\mathrm{CH}, \mathrm{E}_{\text {Dat }}$ is assigned a value 0 . In practice, $E_{\text {Trans }}=0$ signifies inefficiency of the clustering method and hence can't assure energy conservation or even successful transmission. Considering it as a key motivation, we derived a performance parameter called "Efficiency", which was estimated using, given in (15).

Efficiency $=\frac{(\text { Number of connected node })}{\mathrm{E}_{\text {Trans }}}$

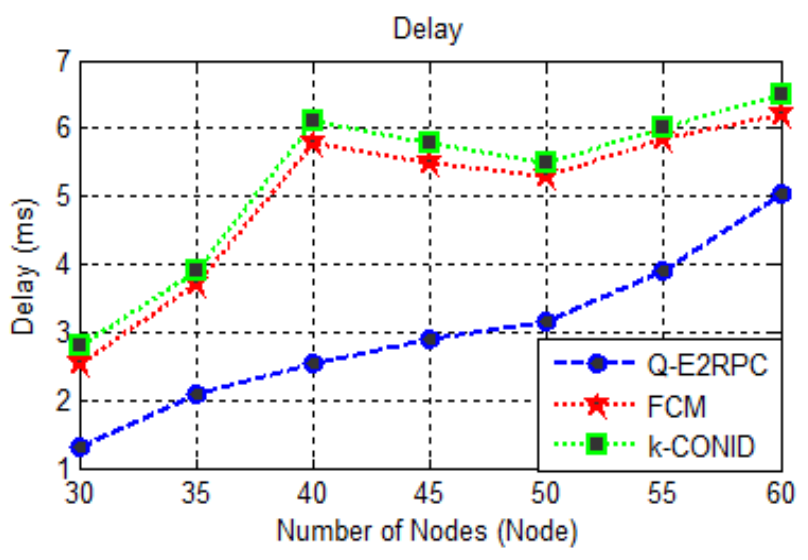

Fig. 12 Transmission or data gathering delay

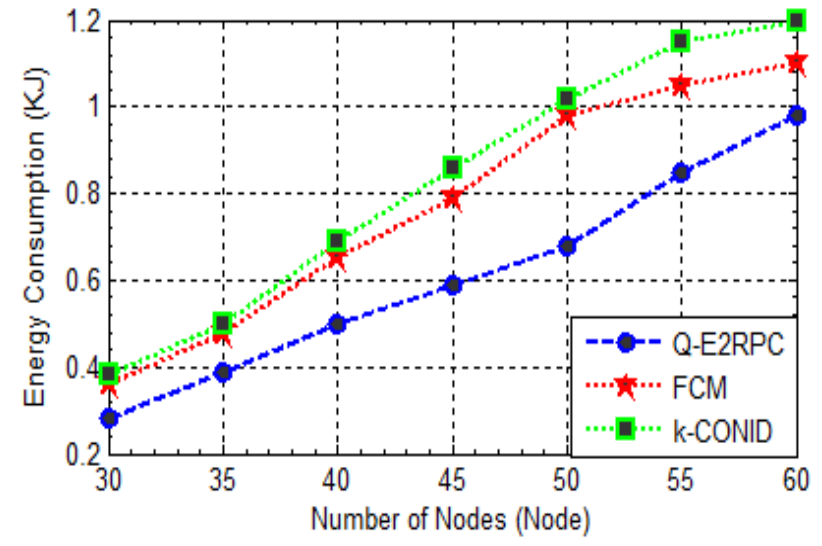

Fig. 13 Energy consumption by different techniques

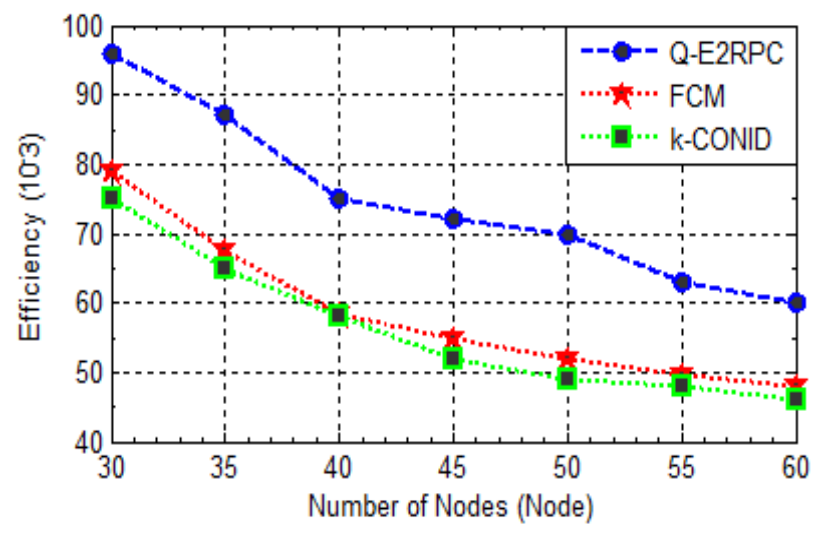

Fig. 14 Network communication efficiency

\section{Research Question Reasoning}

Recalling the research questionnaires (Section III) and the results obtained it can be easily observed that the use of multiple network parameters such as, residual energy, link quality or SNR, distance, and node responsiveness can play vital role in the optimal $\mathrm{CH}$ selection. Undeniably, some of the node parameters change over simulation period (For Example, signal to noise ratio vary over channel conditions, mobility too can vary network topology and hence inter-node distance). Therefore the use of these parameters for $\mathrm{CH}$ selection can strengthen fault resilient $\mathrm{CH}$ selection. This can lead reliable transmission over WSN. This research work also exhibited that the use of FLC can perform better to learn multiple parameters and can help in deciding swift $\mathrm{CH}$ selection. Exploring initial phase of clustering the use of FCM in conjunction of EM has enabled optimal clustering. This can be due to the use of responsiveness or degree of dependence of a node on a cluster. It shows that a node with higher dependence and responsiveness night often intend to support reliable transmission without undergoing high traversal and drop-probability. Furthermore, this research work and resulting outcomes have revealed that unlike multihop transmission based approaches the use of a single mobile sink can reduce traversal period, energy exhaustion due to retransmission (at multiple hops) and data drop probability. It can play vital role in assuring QoS delivery and energy efficient communication over WSNs. The use of CMIMO for transmission too have played significant role in assuring efficient communication. Thus, the proposed routing protocol, Q-E2RPC as cumulative solution has enabled an optimal routing protocol for QoS oriented and energy efficient communication over WSN. 


\section{Conclusion}

In this paper a robust and efficient mobility assisted WSN routing protocol was developed. To achieve an energy efficient and QoS centric routing model, in this research work the emphasis was made on enhancing the key components such as network partitioning, enhanced clustering, soft-computing based cluster head selection, and CMIMO based transmission. The overall results obtained in terms of packet delivery ratio, energy consumption, delay and efficiency exhibit that Q-E2RPC outperforms traditional approaches applying CMIMO and single parameter based $\mathrm{CH}$ selection. In future, some other decision algorithms such as Neural Network or Evolutionary computing based approaches can be explored to assist clustering and $\mathrm{CH}$ selection.

This is an Open Access article distributed under the terms of the Creative Commons Attribution License

\section{References}

[1] Akyildiz, I., Su, W., Sankarasubramaniam, Y., and Cayirci, E. "Wireless sensor networks: a survey". Computer Networks, vol. 38, 393-422 (2002).

[2] Dietrich, I., and Dressler, F. "On the Lifetime of Wireless Sensor Networks". ACM Transactions on Sensor Networks. vol. 5 (2009).

[3] Ang, L. M., Seng, K. P., Chew, L. W., Yeong, L. S., and Chia, W. C. "Wireless Multimedia Sensor Networks on Reconfigurable Hardware: Information Reduction Techniques. Springer Science \& Business Media” (2013).

[4] Mini, R. A., and Loureiro, A. A. " Energy in wireless sensor networks. in Middleware for Network Eccentric and Mobile Applications". Springer, 3-24 (2009).

[5] Cho, S., and Chandrakasan, A. "Energy-efficient protocols for low duty cycle wireless microsensor". In Proceedings of the 33rd Annual Hawaii International Conference on System Sciences (2000).

[6] Ganesan, D., Govindan, R., Shenker, S., and Estrin, D. "Highlyresilient, energy-efficient multipath routing in wireless sensor networks". ACM SIGMOBILE Mobile Computing and Communications Review. vol. 5, no. 4, 1125 (2001).

[7] Mietzner, J., Schober, R., Lampe, L., Gerstacker, W. H., and Hoeher, P. A."Multiple-antenna techniques for wireless communications-a comprehensive literature survey". Communications Surveys \& Tutorials, IEEE, vol. 11, no. 2, 87105(2009).

[8] Laneman, J. N., Tse, D. N. C., and Wornell, G. W. "Cooperative diversity in wireless networks: Efficient protocols and outage behaviour". IEEE Transactions on Information Theory, vol. 50, no. $12,3062-3080$ (2004).

[9] Sendonaris, A., Erkip, E., and Aazhang, B."User cooperation diversity-part I: System description". IEEE Transactions on Communications, vol. 51, no. 11, 1927-1938 (2003).

[10] Sendonaris, A., Erkip, E., and Aazhang, B."User cooperation diversity-part II: Implementation aspects and performance analysis," IEEE Transactions on Communications, vol. 51, no. 11, 1939-1948 (2003).

[11] He, P., Tian H., and Shen, H. "Energy-efficient cooperative MIMO routing in wireless sensor networks". 18th IEEE International Conference on Networks (ICON), Singapore, 74-79 (2012).

[12] Heinzelman, W. R., Chandrakasan, A., and Balakrishnan, H. “An application-specific protocol architecture for wireless microsensor networks". IEEE Transactions on Wireless Communications, vol. 1, no. 4, 660-670 (2002).

[13] Qin, Y., Tang, Q., Liang, Y., Yue X., and Li, X. "An EnergyEfficient Cooperative MIMO Scheme for Wireless Sensor Networks Based on Clustering". 14th IEEE International Conference on Computational Science and Engineering, Dalian, Liaoning, 471-474 (2011).

[14] Singh, Debabrata, and Sanjeet Kumar Nayak. "Enhanced modified LEACH (EMODLEACH) protocol for WSN." Advanced Computing and Communication (ISACC), 2015 International Symposium on. IEEE, 2015.

[15] Xiong, Z., Chen, W., and Cao, W. " An Energy-Efficient ClusterBased Cooperative MIMO Scheme Using Network Coding”. 7th International Conference on Wireless Communications, Networking and Mobile Computing, Wuhan. 1-5 (2011).

[16] Singh, Debabrata, Binod Kumar Pattanayak, and Chandan Kumar Panda. "Analysis of an improved energy balanced routing protocol for wireless sensor network." Communication and Signal Processing (ICCSP), 2016 International Conference on. IEEE, 2016.

[17] Singh, Debabrata, and Binod Kumar Pattanayak. "Analytical Study of an Improved Cluster based Routing Protocol in Wireless Sensor Network." Indian Journal of Science and Technology 9.37 (2016).

[18] Fei, L., Gao Q., and Zhang, X. "Energy efficient cooperative MIMO with Idle nodes in cluster based wireless sensor networks". 6th International Symposium on Wireless Comm. Systems, Tuscany, 448-452 (2009).

[19] Siam, M. Z., Krunz M., and Younis, O. "Energy-Efficient Clustering/Routing for Cooperative MIMO Operation in Sensor Networks". IEEE INFOCOM, Rio de Janeiro, 621-629 (2009).

[20] Islam, M. R., and Kim, J. "Energy efficient cooperative MIMO in wireless sensor network". International Conference on Intelligent Sensors, Sensor Networks and Information Processing, Sydney, NSW, 505-510 (2008).

[21] Cheng, W., Xu, K., Liu, W., Yang Z., and Feng, Z. "An EnergyEfficient Cooperative MIMO Transmission Scheme for Wireless Sensor Networks". International Conference on Wireless Communications, Networking and Mobile Computing, Wuhan, 1-4 (2006)

[22] Shuguang Cui, and Goldsmith, A. J. "Energy efficient routing based on cooperative MIMO techniques. Proceedings". (ICASSP '05). IEEE International Conference on Acoustics, Speech, and Signal Processing, vol. 5, v/805-v/808 (2005).

[23] Gao, T., Zhang, L., Gai Y., and Shan, X. 2007. Load-Balanced Cluster-Based Cooperative MIMO Transmission for Wireless Sensor Networks. 4th International Symposium on Wireless Communication Systems, Trondheim, 602-606 (2007).

[24] Pillutla L. S., and Krishnamurthy, V."Joint rate and cluster optimization in cooperative MIMO sensor networks". IEEE 6th Workshop on Signal Processing Advances in Wireless Communications, 265-269 (2005).

[25] Li, B., Li, H., Wang, W., Yin Q., and Liu, H. "Performance Analysis and Optimization for Energy-Efficient Cooperative Transmission in Random Wireless Sensor Network". In IEEE Transactions on Wireless Communications, vol. 12, no. 9, 46474657 (2013).

[26] Alami Chaibrassou, Ahmed Mouhsen. "An Energy Efficient Cooperative MIMO Routing Protocol for Cluster Based WSNs". Proceedings of the Mediterranean Conference on Information \& Communication Technologies, 229-237 (2015).

[27] Chaibrassou A., and Mouhsen, A. "A multi-channel cooperative MIMO routing protocol for clustered WSNs". International Conference on Electrical and Information Technologies (ICEIT), Tangiers, 231-236 (2016).

[28] Nguyen, D. N., Marwan Krunz. "A Cooperative MIMO Framework for Wireless Sensor Networks". ACM Transactions on Sensor Networks (TOSN), vol. 10, no. 3 (2014).

[29] Sharmila, D., Sujitha, R., and Rajkumar G. "On Improving the Lifetime of Wireless Sensor Networks Using Virtual Scheduling Backbone Replacement". Proceedings of IEEE Conference on Information and Communication Technologies(2013).

[30] Jaafar, W., Ajib, W., and Haccoun, D. "On the performance of distributed-STBC in multi-hop wireless relay networks". in Proceedings of the European Wireless Conference, 223-230 (2010).

[31] Jayaweera, S. "Virtual MIMO-based cooperative communication for energy-constrained wireless sensor networks". IEEE 
Kummathi Chenna Reddy, Geetha D Devanagavi and Thippeswamy MN/

Journal of Engineering Science and Technology Review 12 (1) (2019) 213 - 224

Transactions on Wireless Communications, vol. 5, no. 5, 984989 (2006).

[32] Yong Yuan, Min Chen, Taekyoung Kwon.”A novel cluster-based cooperative MIMO scheme for multi-hop wireless sensor networks" EURASIP Journal on Wireless Communications and Networking, vol. 2006, no. 2, 38-38 (2006).

[33] Naga Malleswari P., Venkateswara Rao, G. "An Energy Efficient Virtual MIMO Communication for Cluster-based Wireless Sensor Networks". In International Journal of Engineering Research \& Technology (IJERT), Vol. 2, no. 4, 822-825 (2013).

[34] Md. Abdullah-al Mamun, Naoshi Nakaya, Yuji koi, Yukari Hagihara. "HACC: Heterogeneous-Aware Cooperative Clustering Hierarchy for wireless Sensor Networks". In IEEE, 1113-1118 (2011).

[35] Vibhav Kumar Sachan, Syed A. Imam, and Beg, M.T. "Energyefficiency of Virtual Cooperative MIMO Techniques in Wireless Sensor Networks". In international Conference on Computer Communication and Informatics (2012).

[36] Neeraj Tiwari, Rahul Anshumali, Prabal Pratap Singh."Energy Efficient Virtual Mimo-Based Cooperation Communication in Wire-Less Sensor Networks". In International Journal of Advanced Technology \& Engineering Research (IJATER), vol. 2, no. 3, 53-58 (2012).

[37] Li Wang, Yuhan Dong, and Xuedan Zhang."Throughput Maximization Transmission Scheme for Virtual MIMO in Clustered Wireless Sensor Networks". In Wireless Communications and Networking Conference (WCNC), IEEE, 3632-3636 (2013).

[38] Jie Ding, Danpu Liu, Xin Wang and Huari Wu. "An energyefficient virtual MIMO transmission scheme for cluster-based wireless sensor networks". IEEE 12th International Conference on Communication Technology, Nanjing, 809-813 (2010).

[39] Xu, K., Yuan, W., Cheng, W., Ding, Y., and Yang, Z. "An Energy-Efficient V-BLAST Based Cooperative MIMO Transmission Scheme for Wireless Sensor Networks". IEEE Wireless Communications and Networking Conference, Las Vegas, NV, 688-693 (2008).

[40] Medhia, N., Sarmab, N."Mobility Aided Cooperative MIMO Transmission in Wireless Sensor Networks". 2nd International Conference on Communication, Computing \& Security, a Procedia Technology, vol. 6, 362 - 370 (2012).

[41] Gao, Q., Zuo, Y., Zhang, J., and Peng, X. H. "Improving energy efficiency in a wireless sensor network by combining cooperative MIMO with data aggregation". IEEE Transactions on Vehicular Technology, vol. 59, no. 8. 3956-3965 (2010).

[42] Vidhya J., and Dananjayan P."Lifetime Maximisation of Multihop WSN using Cluster-based Cooperative MIMO Scheme". International Journal of Computer Theory and Engineering, vol. 2, no. 1, 1793-8201 (2010).

[43] Cui, S., Goldsmith, A. J., and Bahai, A. "Energy-efficiency of MIMO and cooperative MIMO techniques in sensor networks". IEEE Journal on Selected Areas in Communications, vol. 22, no. 6, 1089-1098 (2004).
[44] Jayaweera, S. K. "Energy analysis of MIMO techniques in wireless sensor networks". In Proceedings of the 38th Annual Conference on Information Sciences and Systems (CISS) (2004).

[45] Sathian, D., Baskaran, R., and Dhavachelvan, P.'Lifetime enhancement by Cluster Head Cooperative Trustworthy Energy Efficient MIMO routing algorithm based on game theory for WSN". Computing Communication \& Networking Technologies (ICCCNT), Third International Conference on, Coimbatore, 1-5 (2012).

[46] Islam, M. R. "Energy efficient cluster to cluster communication for correlated data at wireless sensor network". International Conference on Electrical \& Computer Engineering, Dhaka, 131134 (2010).

[47] Zhang, Y., Cai, Y., Yang W., and Xu, Y. “An Energy-Efficient Cooperative Node Selection Scheme in Wireless Sensor Networks". International Conference on Comm. Software and Networks, Macau. 232-236 (2009).

[48] Navroop kaur Chattha. "energy balancing intelligent clustring protocol for wireless sensor network". Journal of Global Research in Computer Science.

[49] Dabirmoghaddam, A., Ghaderi, M., and Williamson, C. "Energyefficient clustering in wireless sensor networks with spatially correlated data". in Proceedings of the IEEE INFOCOM Conference, 1-2 (2010).

[50] Joshi, M., Osborne, L., Sun, B., and Makki, S. "Hot spot aware energy efficient clustering approach for wireless sensor networks". In Proceedings of the IEEE Consumer Communications and Networking Conference (CCNC), 585-589 (2011).

[51] Enami, N., Moghadam R. A., and Ahmadi, K. D. 2010. “A new neural network based energy efficient clustering protocol for Wireless Sensor Networks". 5th International Conference on Computer Sciences and Convergence Information Technology, Seoul, 40-45 (2010).

[52] Amri, S., Kaddachi, M. L., and Trad, A. "Energy-Efficient Multihop Hierarchical Routing Protocol using Fuzzy Logic (EMHRFL) for Wireless Sensor Networks". World Congress on Computer Applications and Information Systems (WCCAIS), Hammamet, 1-6 (2014).

[53] Kubo, M., Sun, M., Yanagihara K., and Hara,S. "A multiple cooperative nodes selection method for reliable wireless multihop data transmission". International Symposium on Wireless Communication Systems (ISWCS), Paris, 486-490 (2012).

[54] Hong, S. I., Lin, C. H. 2016.”An expansion cluster routing algorithm based on RSSI for an efficient data transmission". In Proceedings of the 2016 18th International Conference on Advanced Communication Technology, PyeongChang, Korea, 31-33 (2016).

[55] Saeed, N., Nam, H. “Cluster based multidimensional scaling for irregular cognitive radio networks localization". IEEE Trans. Signal Process., vol. 64, 2649-2659 (2016).

[56] Geng Chen, Nocetti, F. G., Gonzalez, J. S., and Stojmenovic, I. "Connectivity based k-hop clustering in wireless networks". Proceedings of the 35th Annual Hawaii International Conference on System Sciences, 2450-2459 (2002). 